\title{
4 Sexualität im Alter
}

\author{
Diana Richter, Kristina Geue, Lutz Gansera und Elmar Brähler
}

Auf einem Kongress hat ein Dreißigjähriger zu mir gesagt: „Muss ein Siebzigjähriger wirklich noch Viagra nehmen? Der hat doch schon genug schöne Sachen mitgemacht.“

Ich habe geantwortet: „Okay, gehen Sie zu Ihrem Großvater und sagen Sie ihm: Du brauchst keine Brille mehr, du hast schon genug gesehen."

(Oswalt Kolle, 2007 im Interview für die Stuttgarter Zeitung)

\subsection{Einleitung}

Ältere Paare und Geschlechtsverkehr - eine Vorstellung, die für die Mehrzahl der jüngeren „Anti-Aging“ Generationen nicht mit ihrem Bild der „Alten“ zu vereinbaren ist. Sexualität ist hauptsächlich den jungen und gesunden Menschen vorbehalten (Bamler 2008).

Das eher defizitorientierte Altersbild spricht älteren Menschen eine Sexualität ab. Sexualität im Alter stellt zudem immer noch ein Tabuthema in der Gesellschaft dar. Und dies nicht nur, weil die Jüngeren nicht mit der älteren Generation darüber sprechen, sondern auch, weil die meisten älteren Menschen der heutigen Zeit es nicht anders gelernt haben. Wer heute zu den alternden Menschen ab 50 gehört, wurde anders sozialisiert; eine offene Kommunikation über Sexualität war und ist tabu (Merbach et al. 2003).

Dabei kommen gravierende Veränderungen der Altersstruktur auf uns zu: Laut statistischem Bundesamt wird 2060 jeder Dritte mindestens 65 Jahre alt, jeder siebente wird 80 Jahre und älter sein. Dies entspricht etwa dem Anteil der unter 20-Jährigen. Mit einer steigenden Lebenserwartung verstetigt sich auch der Wunsch nach möglichst langer Gesundheit und Aktivität bis ins hohe Alter. Eine gute Gesundheit wiederum korreliert mit einer höheren Sexrate und sexueller Befriedigung bei älteren Menschen (Yee 2010). In jüngster Zeit rückt das Kompetenzmodell des „Erfolgreichen Alterns“ nach Baltes immer mehr in den Vordergrund, welches sich in einigen Studien widerspiegelt, deren Gegenstand das sexuelle Erleben im Alter ist. 


\section{Sexualität im Alter ist in unserer Gesellschaft ein Tabuthema.}

\subsection{Einflussfaktoren auf die Sexualität}

Die Sexualität vom älter werdenden Mann wird durch mehrere Faktoren beeinflusst.

\subsubsection{Biologische Einflussfaktoren}

Mit zunehmendem Alter treten typische Beeinträchtigungen der sexuellen Funktion auf. Diese hängen im Wesentlichen vom allgemeinen Gesundheitszustand ab, denn die Wahrscheinlichkeit von Begleiterkrankungen wie Diabetes, Morbus Parkinson etc. steigt linear zum Alter an. Aber auch (die daraus resultierenden) Nebenwirkungen der Medikamenteneinnahme können die sexuelle Aktivität stark beeinträchtigen (DeLamater u. Moorman 2007).

Eine hormonelle Veränderung manifestiert sich ab einem Alter von 40 Jahren. Der Testosteronspiegel des Mannes sinkt nun um etwa 1\% pro Jahr. Bei einigen Männern führt ein starker Testosteronmangel zu mehreren Symptomen. Für diese endokrinologischen Veränderungen hat sich die Bezeichnung „PADAM“ (partielles Androgendefizit des alternden Mannes) verbreitet (Ludwig 2001). Ab 65 Jahre treten erste degenerative Veränderungen in den Samenkanälchen des Hodens auf (Bucher et al. 2001). Die Spermienproduktion nimmt ab, die Spermienqualität wird schlechter und das Volumen des Ejakulats wird kleiner. Auch Marandola et al. (2002) sprechen von einem veränderten sexuellen Reaktionszyklus im höheren Lebensalter, weshalb Männer mehr Zeit brauchen, eine Erektion zu erreichen und diese länger zu halten, Orgasmen werden kürzer und die Refraktärphase nimmt deutlich zu. Trotz dieser körperlichen Veränderungen, die jeder Mann unterschiedlich wahrnimmt, bleibt die Zeugungsfähigkeit bis ins hohe Alter erhalten. Carpenter et al. (2006) konnten in einer Studie bestätigen, dass die Auswirkungen des Alterns eher die Frauen betreffen und vor allem auch gravierender sind als bei den Männern.

\subsubsection{Psychosoziale Einflussfaktoren}

Ab einem Alter von etwa 50 Jahren kommt es in vielen Lebensbereichen zu einschneidenden und destabilisierenden Veränderungen (bspw. durch den Auszug der Kinder), welchen sich ältere Männer schneller anpassen können als in jüngerem Alter (Seikowski u. Paasch 2010).

Sexualität wird in allen Altersphasen gelebt. Die individuelle sexuelle Entwicklung orientiert sich an den jeweiligen normativen Vorgaben und vor allem an der sexuellen Biografie (Bamler 2008; Bitzer 2005). Menschen, die im jungen Erwachsenenalter sexuell aktiv waren, bleiben dies auch bis ins höhere Alter. Negative sexuelle Erfahrungen sind dagegen hinderlich. Ebenso können Schamgefühle im Zusammenhang mit sexuellem Versagen zu einer manifesten Versagensangst führen (Bitzer 2005).

Eine wichtige Rolle spielt auch das Vorhandensein eines sexuell interessierten Partners. Dies betrifft besonders Frauen, die durch das zeitigere Versterben ihres Partners 
verwitwet sind und of tmals ohne neuen Partner ihren Lebensabend verbringen und ihre sexuellen Bedürfnisse nicht stillen können (Bamler 2008).

Aus diesem Komplex an Einflussfaktoren kann konstatiert werden, dass sich die Sexualität im Alter neu definieren muss. Mit zunehmendem Alter ist die Sexualität von einer anderen Motivation getragen „Es ist weniger die Lust, am Geschlechtsverkehr selbst, als die Lust am anderen Menschen, dem ich mit Zärtlichkeit Gefühle mitteilen kann [ ]“ (Zapotoczky 2003). Sexualität verliert ihre reproduktive Funktion und wird vielmehr als Kommunikationsform und zum Stillen der Lustbefriedigung relevanter.

\section{Der Einfluss auf die Sexualität im Alter ist multifaktoriell.}

\subsection{Sexuelles Verlangen}

Studien zur Sexualität betonen vor allem die Geschlechterunterschiede. Bereits bei der Frage nach dem Stellenwert von Sex werden signifikante Unterschiede sichtbar. So zeigten Waite et al. (2009), dass etwa 52\% der Frauen zwischen 75 und 85 Jahren Sex als „nicht wichtigen Teil des Lebens“ einstufen, dagegen nur etwa 26\% der Männer in dieser Altersgruppe. Die Frage „Ob und wie sich das sexuelle Interesse im mittleren und höheren Erwachsenenalter verändert?" wurde in der Studie von Bucher und Kollegen (2001) thematisiert. Der Wunsch nach Geschlechtsverkehr nahm erst bei den über 70-jährigen Männern ab, wohingegen bei den Frauen dieser Trend schon ab 65 Jahren auftrat und im Vergleich zu den Männern etwas deutlicher ausfällt. Die Diskrepanz zwischen den Wünschen und der Realität bezüglich des Geschlechtsverkehrs wird mit zunehmendem Alter größer. Zärtlichkeiten wünschen sich Männer bis ins hohe Alter, bei den Frauen nimmt dieser Wunsch bei den über 7o-jährigen leicht ab.

Fazit ist, dass es keine bedeutsamen Unterschiede in dem Wunsch nach Zärtlichkeit, Petting, Geschlechtsverkehr sowie sexuellen Gedanken, Fantasien und Träumen von Männern im mittleren und höheren Alter gibt. Beutel et al. (2008) kommen in ihrer repräsentativen Untersuchung zu dem Schluss, dass das sexuelle Verlangen mit zunehmendem Alter insgesamt abnimmt, jedoch gaben die Männer ein häufigeres und stärkeres sexuelles Verlangen im Gegensatz zu den Frauen an. Die Prävalenz des mangelnden sexuellen Verlangens schwankt bei Frauen zwischen 10 bis 41\%, bei Männern zwischen 2 bis 15\% (Beutel et al. 2009). DeLamater und Moorman (2007) konnten bestätigen, dass jüngere Menschen eine variantenreichere Sexualität ausleben. Ältere Menschen bevorzugen vaginalen Sex und das Vorspiel. Dies bleibt bei den ,jüngeren Alten“ so konstant wie bei den „alten Alten“; dagegen sinkt das Verlangen nach Oralsex und Masturbation (Waite et al. 2009). Die unterschiedliche Wichtigkeit von Sexualität zeigt sich am deutlichsten bei den über 8o-Jährigen: Bei Frauen sinkt diese viel stärker als bei Männern (Beutel et al. 2009). Das Interesse an sexueller Aktivität bleibt für die Mehrheit der Männer bis ins hohe Lebensalter bestehen. 


\subsection{Sexuelle Aktivität}

Im höheren Lebensalter wünschen sich Männer mehr sexuelle Aktivität als Frauen (Gunzelmann et al. 2004). So masturbiert etwa jeder zweite über 65-jährige Mann. Die repräsentative Erhebung von Klaiberg et al. (2001) zeigt auf, dass die Mehrzahl der älteren Männer (75\%) im letzten Jahr intimen Kontakt hatte, wobei dies für 83\% der Männer mit Partnerin zutrifft und für 59\% der Männer ohne Partnerin. Ebenso wie das sexuelle Verlangen nimmt auch die sexuelle Aktivität mit zunehmendem Alter ab (Beutel et al. 2009). In der Befragung von Beutel et al. (2008) nahm die sexuelle Aktivität bei den über 6o-jährigen Männern deutlich ab, bei den Frauen jedoch in viel größerem Ausmaß. Festzuhalten ist, dass die Partnerschaft einen großen Einfluss auf die sexuelle Aktivität hat. So ist nur etwa jeder sechste Mann, der nicht in einer Partnerschaft lebt, sexuell aktiv. Weitere Einflussgrößen waren das Alter und der Wohnort (Stadt vs. Land). Die Bildung, die Konfession und das Einkommen waren für die sexuelle Aktivität nicht bedeutsam. Als Gründe für die sexuelle Inaktivität fand Bucher (2009) heraus: keine Partnerin, Partnerin kann nicht mehr, keine Lust, Partnerin will nicht mehr und/oder die eigene Impotenz. Obwohl das Altern zu Veränderungen im sexuellen Reaktionszyklus des Mannes führt (Marandola et al. 2002), hat dies geringere Auswirkungen auf die sexuelle Aktivität als bisher angenommen. Hierfür spricht vor allem der Zusammenhang zwischen sexueller Aktivität und einer bestehenden Partnerschaft. Es sollte auch berücksichtigt werden, dass ein Partner im Geschlechtervergleich unterschiedlich verfügbar ist: Männer sind zum Großteil bis ins hohe Alter verheiratet, bei Frauen sinkt dies und der Anteil der verwitweten Frauen steigt (Beutel et al. 2009).

\subsection{Sexuelle Zufriedenheit}

Im Vergleich zu Jüngeren kommt es mit zunehmendem Alter zu einer leichten Abnahme der sexuellen Zufriedenheit, wohingegen die Zufriedenheit mit der Partnerschaft leicht ansteigt (Klaiberg et al. 2001). Interessant ist, dass Männer angeben, sowohl mit der Sexualität als auch der Partnerschaft zufriedener zu sein im Vergleich zu den Frauen. Auf das Alter bezogen sind unter den Männern die jüngeren Älteren mit ihrer Sexualität zufriedener. Ältere Menschen, die mehr sexuelle Kontakte hatten, in einer Partnerschaft lebten und einen besseren Gesundheitszustand angaben, beurteilten ihre Sexualität besser. Ähnlich wie mit dem sexuellen Verlangen verhält es sich mit der sexuellen Zufriedenheit: Der Abfall der Zufriedenheit unterscheidet sich zwischen den Geschlechtern bei den über 8o-Jährigen am deutlichsten (Beutel et al. 2009). Vor allem über 8o-jährige Frauen sind wesentlich unzufriedener. Wichtige Einflussfaktoren sind vor allem das Vorhandensein einer Partnerschaft, ein höheres Haushaltseinkommen und der Wohnort in den alten Bundesländern (Beutel et al. 2009).

\section{Fazit}

Ab der Lebensmitte verändert sich die Sexualität der Männer durch mehrere Determinanten. Wie stark dies Männer beeinflusst, ist individuell verschieden.

Sexuelle Probleme treten weitaus häufiger im höheren Lebensalter auf, tauchen aber dagegen selten im Arzt-Patienten-Gespräch auf. 


\subsection{Praktische Relevanz}

In der Praxis sollte dieses komplexe Zusammenwirken vor allem in der Diagnosefindung als auch in Beratungen berücksichtigt werden. Ältere Menschen finden es angemessen, mit ihrem Arzt über sexuelle Probleme zu sprechen, aber genieren sich. Deshalb sollten Ärzte wertungsfrei mit ihren Patienten über Sexualität reden und sich über das ganze Spektrum an gesundheitsbezogenen, möglicherweise behandelbaren sexuellen Problemen bewusst sein (Carpenter et al. 2006). Demgegenüber steht der Mangel an speziellen Beratungsangeboten für Ältere (Schultz-Zehden 2004). Um dies langfristig zu installieren, bedarf es einer Enttabuisierung von Sexualität im Alter, indem Gesprächsbarrieren abgebaut werden und die Vorstellung der Asexualität im Alter verworfen wird.

\section{Exkurs - Partnerschaft und Sexualität in Senioreneinrichtungen}

Viele Senioren führen bis ins hohe Alter ein selbstbestimmtes Leben in den eigenen vier Wänden. Für andere, die im fortgeschrittenen Alter nicht mehr allein wohnen können oder wollen, kann der Wechsel in eine Senioreneinrichtung eine Alternative darstellen. Laut Schneider (1998) waren 8\% der Bewohner in Alten- und Pflegeheimen verheiratet, $67 \%$ verwitwet, $5 \%$ geschieden und rund $20 \%$ ledig.

Das Thema Partnerschaft und Sexualität gehört - auch wenn den in Alten- und Pflegeeinrichtungen lebenden Menschen das Ausleben ihrer Sexualität lange Zeit abgesprochen oder sogar verboten wurde - zu den basalen physiologischen Bedürfnissen des Menschen. So praktizieren sexuell Interessierte vielfältige Handlungsweisen, welche von sexueller Abstinenz bis hin zum Ausleben von Zärtlichkeiten und sexuell-genitalen Aktivitäten reichen können. Die institutionellen Rahmenbedingungen schränken jedoch erotische Kontakte durch die fehlende Intimsphäre oft erheblich ein (Betreten der Zimmer ohne Anklopfen, Mehrbettzimmer etc.). Zudem tragen das infantile Anreden der Bewohner sowie das mehr oder weniger geschmackvolle „Aufhübschen“ der Bewohner mit Schleifchen, Zöpfchen etc. zur Desexualisierung der Bewohner bei. Mitunter stoßen Bewohner, die ihre Zuneigung zueinander bekennen, nicht nur beim Personal, sondern auch bei den Mitbewohnern auf Unverständnis bis Ablehnung.

Der adäquate Umgang mit sexueller Enthemmung (z.B. bei Demenzkranken) verlangt vom Pflegepersonal eine hohe fachliche und menschliche Kompetenz. Zu schwierigen bis konfliktgeladenen Situationen kann es jedoch auch kommen, wenn Pflegende durch körperliche Übergriffe, verbale Äußerungen oder durch das Ausnutzen von pflegerischen Verrichtungen zum Zwecke sexueller Stimulation belästigt werden (Schroeter u. Prahl 1999). Gezielte Weiterbildungen könnten hier ansetzen.

Angesichts der im Alter abnehmenden sozialen Kontakte ist es nicht zuletzt auch im Sinne der Senioreneinrichtungen, diese zu fördern und ein selbstbestimmtes Leben zu ermöglichen, zumal Liebe, Sex und Partnerschaft keine Vorrechte einer Altersgruppe sind.

\section{Ein vertrautes Miteinander braucht (einen geschützten) Raum und Zeit.}




\section{Literatur}

Bamler V (2008) Sexualität in der dritten Lebensphase. In: Buchen S, Maier MS (Hrsg.) Älterwerden neu denken. Interdisziplinäre Perspektiven auf den demographischen Wandel. Wiesbaden, VS Verlag für Sozialwissenschaften, 181-195

Beutel ME, Siedentopf F, Brähler E (2009) Sexuelle Aktivität und Zufriedenheit mit Sexualität und Partnerschaft im Alter. In: Brähler E, Berberich H (Hrsg.) Sexualität und Partnerschaft im Alter. Gießen, Psychosozial, 27-44

Beutel ME, Stöbel-Richter Y, Daig I, Brähler E (2008) Sexuelles Verlangen und sexuelle Aktivität von Männern und Frauen über die Lebensspanne - Ergebnisse einer repräsentativen deutschen Bevölkerungsumfrage. I Reproduktionsmed Endokrinol 4, 203-211

Bitzer I (2005) Die Sexualität des älter werdenden Paares. Journal für Menopause 12, 6-9

Bucher T (2009) Altern und Sexualität. In: Brähler E, Berberich H (Hrsg.) Sexualität und Partnerschaft im Alter. Gießen, Psychosozial, 45-63

Bucher T, Hornung R, Gutzwiller F, Buddeberg C (2001) Sexualität in der zweiten Lebenshälfte. Erste Ergebnisse einer Studie in der deutschsprachigen Schweiz. In: Berberich H, Brähler E (Hrsg.) Sexualität und Partnerschaft in der zweiten Lebenshälfte. Gießen, Psychosozial 31-59

Carpenter LM, Nathanson CA, Kim YJ (2006) Sex After 40?: Gender, Ageism, and Sexual Partnering in Midlife. Journal of Aging Studies 20, 93-106

DeLamater I, Moorman SM (2007) Sexual behavior in later life. Journal of Aging and Health 19, 921-945

Gunzelmann T, Rusch BD, Brähler E (2004) Einstellungen zu Erotik und sexueller Aktivität bei über 60-Jährigen. Gesundheitswesen 66, 15-20

Klaiberg A, Brähler E, Schumacher I (2001) Determinanten der Zufriedenheit mit Sexualität und Partnerschaft in der zweiten Lebenshälfte. In: Berberich H, Brähler E. Sexualität und Partnerschaft in der zweiten Lebenshälfte. Gießen, Psychosozial 105-127

Ludwig G (2001) Endokrine Veränderungen beim älteren Mann aus urologischer Sicht. In: Berberich H, Brähler E (Hrsg.) Sexualität und Partnerschaft in der zweiten Lebenshälfte. Gießen, Psychosozial 159-165

Marandola P, Musitelli S, Noseda R, Stancati S, Vitetta D, Jallous H, Porru D, Zmerly H, Mensi M, Colombo P, Orlando G, Cebrelli T, Gerardini M, Giliberto G, Pasini L, Lillaz B, Pietropaolo F, Sali C, Rovereto B (2002) Love and sexuality in aging. The Aging Male 5, 103-113

Merbach M, Beutel M, Brähler E (2003) Sexualität des alternden Mannes. BZgA Forum Sexualaufklärung und Familienplanung. Alter und Sexualität. Informationsdienst der Bundeszentrale für gesundheitliche Aufklärung, 1/2, 7-11

Schneider S (1998) Ältere Bundesbürger in Privathaushalten und in Heimen. Lebenssituation und Heimeintrittsgründe. Sozialer Fortschritt 2, 30-37

Schroeter KR, Prahl HW (1999) Soziologisches Grundwissen für Altenhilfeberufe. Beltz Verlag

Schultz-Zehden B (2004) Wie wandelt sich Sexualität im Alter? Das Sexualleben älterer Frauen - ein tabuisiertes Thema. fundiert 1, 50-55

Seikowski K, Paasch U (2010) Der Alternde Mann. In: Bardehle D, Stiehler M (Hrsg.) Erster Deutscher Männergesundheitsbericht. München, W. Zuckerschwerdt Verlag, 58-70

Waite LI, Laumann EO, Das A, Schumm LP (2009) Sexuality: Measures of Partnerships, Practices, Attitudes, and Problems in the National Social Life, Health, and Aging Study. Journals of Gerontology: Series B, 64B, issue suppl_1, i56-i66

Yee L (2010) Aging and Sexuality. Australian Family Physician 39, 705-800

Zapotoczky HG (2003) Sexualität im Alter aus der Sicht des Psychiaters. Journal für Menopause 10, 10-11 